\title{
RADIALLY SYMMETRIC SOLUTIONS TO A DIRICHLET PROBLEM INVOLVING CRITICAL EXPONENTS
}

\author{
ALFONSO CASTRO AND ALEXANDRA KUREPA
}

\begin{abstract}
In this paper we answer, for $N=3,4$, the question raised in [1] on the number of radially symmetric solutions to the boundary value problem $-\Delta u(x)=\lambda u(x)+u(x)|u(x)|^{4 /(N-2)}, x \in B:=\left\{x \in R^{N}:\|x\|<1\right\}, u(x)=$ $0, x \in \partial B$, where $\Delta$ is the Laplacean operator and $\lambda>0$. Indeed, we prove that if $N=3,4$, then for any $\lambda>0$ this problem has only finitely many radial solutions. For $N=3,4,5$ we show that, for each $\lambda>0$, the set of radially symmetric solutions is bounded. Moreover, we establish geometric properties of the branches of solutions bifurcating from zero and from infinity.
\end{abstract}

\section{INTRODUCTION}

We consider the boundary value problem

$$
\begin{gathered}
-\Delta u(x)=\lambda u(x)+u(x)|u(x)|^{p}, \quad x \in B:=\left\{x \in R^{N}:\|x\|<1\right\}, \\
u(x)=0, \quad x \in \partial B,
\end{gathered}
$$

where $\Delta$ denotes the Laplacean operator and $p=\frac{4}{N-2}$. The following theorem answers, for $N=3,4$, the question raised in [1] on the existence of only finitely many radially symmetric solutions to (1.1). Indeed, we prove:

Theorem 1.1. (a) If $N=3,4,5$, then for each $\lambda>0$ there exist positive real numbers $j:=j(\lambda)$ and $D:=D(\lambda)$ such that if $u$ is a solution to (1.1) then $\|u\|_{\infty} \leq D$, and $u$ has at the most $j$ nodal curves.

(b) Given $j$ there exists $D_{1}(j)$ such that if $\left(\lambda_{1}, u_{1}\right)$ and $\left(\lambda_{2}, u_{2}\right)$ are radial solutions to (1.1) with the property that $u_{1}$ and $u_{2}$ have $j$ nodal surfaces, $u_{1}(0)>D_{1}(j)$ and $u_{2}(0)>D_{1}(j)$ then $\lambda_{1}<\lambda_{2}$ if and only if $u_{1}(0)>u_{2}(0)$.

(c) For $N=3,4$, and $\lambda>0$, the boundary value problem (1.1) has finitely many radially symmetric solutions.

Parts (a) and (b) also hold for $N=6$. Since for $N=6$ and $\lambda$ fixed the solutions to (1.1) do not tend to zero in compact sets as $u(0) \rightarrow \infty$, the proof of this case requires different arguments and we defer it to a separate paper.

If $N \geq 7$ then for any $\lambda>0$ it has been proven by Solimini in [12] (see also [6]) that (1.1) has infinitely many radially symmetric solutions. Motivated by this result Atkinson, Brezis and Peletier in [1] studied the case $N=3,4,5,6$

Received by the editors October 20, 1992 and, in revised form, March 11, 1993.

1991 Mathematics Subject Classification. Primary 35J65, 34A10.

Key words and phrases. Critical exponent, radially symmetric solutions, Dirichlet problem, nodal curves, bifurcation. 
and conjectured that problem (1.1) has only finitely many radially symmetric solutions.

Problems like (1.1) have attracted a great deal of attention in recent years, mainly due to the fact that the well-developed variational techniques do not apply because the imbedding of the Sobolev space $H_{0}^{1}(B)$ in $L^{2 N /(N-2)}(B)$ is not compact. Since 1965 it has been known (see Pohozaev [9]) that for $\lambda \leq 0$ the problem (1.1) has no nontrivial solutions. In 1982 Brezis and Nirenberg (see [2]) proved that for certain values of $\lambda>0$ the problem (1.1) has a positive solution. Pursuing the ideas of [2] a number of results have been derived in an attempt to understand why the so-called Palais-Smale condition fails (see $[5,6]$ and the references therein).

The proof of Theorem 1.1 is based on the phase-plane analysis of the solution corresponding to a singular ordinary differential equation. We consider the initial value problem

$$
\begin{aligned}
v^{\prime \prime}(t)+\frac{N-1}{t} v^{\prime}(t)+\lambda v(t)+v(t)|v(t)|^{p} & =0, \quad t \in(0,1], \\
v(0) & =d, \quad v^{\prime}(0)=0
\end{aligned}
$$

where $d \in R$. Arguments based on the contraction mapping principle show that for every $(\lambda, d)$ problem $(1.2)$ has a unique solution $v(t):=v(t, \lambda, d)$ on the interval $[0, \infty)$ depending continuously on $(\lambda, d)$. Of course, radially symmetric solutions to (1.1) are solutions to (1.2) satisfying $v(1, \lambda, d)=0$. Because $v$ is odd in $d$, we consider only the case $d>0$. Thus, we concentrate our analysis on the study of the level set $M=\{(\lambda, d): v(1, \lambda, d)=0\}$. Using the fact that the solution to (1.2) does not degenerate and some rescaling properties of $v(t)$ (see $(3.5))$ we show that $\left(v_{d}(1, \cdot, \cdot), v_{\lambda}(1, \cdot, \cdot)\right)$ never vanishes on $M$, where $v_{d}, v_{\lambda}$ denote the partial derivatives of $v$ with respect to $d$ and $\lambda$. Hence, $M$ is a differentiable manifold. Moreover, using variants of the Sturm comparison theorem we prove that for $d$ large $v_{d}(1, \lambda, d) \cdot v^{\prime}(1, \lambda, d)>0$. Thus if $\Gamma$ is a connected component of $M$ then there exists a strictly decreasing function $s:(D, \infty) \rightarrow R$ such that $v(1, \lambda, d) \in \Gamma$ iff $d=s(\lambda)$. We combine this result with those of [1] to provide a detailed description of the branches of solutions to (1.1) bifurcating from zero and from infinity.

Our proof of the case $N=3,4$, relies on the fact that $v(1, \lambda, \cdot)$ is an analytic function on $(0, \infty)$ (see Appendix), and that solutions to $v(1, \lambda, d)=$ 0 do not accumulate near $d=+\infty$ for fixed $\lambda$.

\section{ANALYSIS OF $v$}

The following lemma is based on Pohozaev's identity. In [3] and [4] this identity was extensively used in the study of subcritical boundary value problems (see also [10]).

Lemma 2.1. If $0 \leq \tilde{t}<t$ then

$$
t^{N-1} H(t)-(\tilde{t})^{N-1} H(\tilde{t})=\int_{\tilde{t}}^{t} r^{N-1} \lambda v^{2}(r) d r
$$


where

$$
\begin{aligned}
H(t) & :=t\left(\frac{\left(v^{\prime}(t)\right)^{2}}{2}+\frac{|v(t)|^{p+2}}{p+2}+\lambda \frac{|v(t)|^{2}}{2}\right)+\frac{N-2}{2} v(t) v^{\prime}(t) \\
& :=t E(t)+\frac{N-2}{2} v(t) v^{\prime}(t) .
\end{aligned}
$$

Proof. Multiplying the equation in (1.2) by $r^{N} v^{\prime}(r)$ and integrating over $[\tilde{t}, t]$, $0 \leq \tilde{t}<t$, we obtain

$$
\begin{aligned}
t^{N} E(t)= & (\tilde{t})^{N} E(\tilde{t}) \\
& -\int_{\tilde{t}}^{t}\left\{\frac{N-2}{2} r^{N-1}\left(v^{\prime}(r)\right)^{2}-N r^{N-1}\left[\frac{\lambda v^{2}(r)}{2}+\frac{|v(r)|^{p+2}}{p+2}\right]\right\} d r .
\end{aligned}
$$

Similarly, multiplying the equation in (1.2) by $r^{N-1} v(r)$ and integrating over $[\tilde{t}, t]$ we infer

$$
\begin{aligned}
\int_{\tilde{t}}^{t} r^{N-1}\left(v^{\prime}(r)\right)^{2} d r= & v^{\prime}(t) v(t) t^{N-1}-v^{\prime}(\tilde{t}) v(\tilde{t})(\tilde{t})^{N-1} \\
& +\int_{\tilde{t}}^{t} r^{N-1}\left[\lambda v^{2}(r)+|v(r)|^{p+2}\right] d r
\end{aligned}
$$

By replacing (2.3) in (2.2), and using the fact that $p=4 /(N-2)$ the lemma follows. From Lemma 2.1 using the quadratic equation formula we see that

$$
\begin{aligned}
t v^{\prime}(t) & +\frac{N-2}{2} v(t) \\
& = \pm \frac{1}{2} \sqrt{(N-2)^{2} v^{2}(t)-\frac{8 t^{2}}{p+2} v^{p+2}(t)-4 \lambda t^{2} v^{2}(t)+8 t^{2-N} \lambda \int_{0}^{t} r^{N-1} v^{2}(r) d r} \\
& := \pm \frac{1}{2} R(t) .
\end{aligned}
$$

Now we define function $h$ by the equation

$$
h(t)=-\frac{t v^{\prime}(t)}{v(t)} .
$$

Using (1.2) and Lemma 2.1 we obtain

$$
\begin{aligned}
h^{\prime}(t) & =\frac{-v(t) v^{\prime}(t)+t v(t)\left\{((N-1) / t) v^{\prime}(t)+\lambda v(t)+|v(t)|^{p} v(t)\right\}+t\left(v^{\prime}(t)\right)^{2}}{v^{2}(t)} \\
& =\frac{(N-2) v(t) v^{\prime}(t)+t \lambda v^{2}(t)+t|v(t)|^{p+2}+t\left(v^{\prime}(t)\right)^{2}}{v^{2}(t)} \\
& =\frac{2 t^{1-N} \int_{0}^{t} \lambda r^{N-1} v^{2}(r) d r-2 t \frac{|v(t)|^{p+2}}{p+2}+t|v(t)|^{p+2}}{v^{2}(t)} \\
& \geq\left(1-\frac{2}{p+2}\right)|v(t)|^{p} t=\frac{2}{N}|v(t)|^{p} t>0 .
\end{aligned}
$$


Let $t_{1}$ denote the first zero of $v$. Since the left-hand side in (2.4) is positive at 0 , negative at $t_{1}$ and continuous, there exists $\hat{t} \in(0, t)$ such that $R(\hat{t})=0$. The uniqueness of $\hat{t}$ follows from (2.6). Since $v^{\prime}(0, \lambda, d)=0$ and $v(0, \lambda, d)=d$, we have

$$
t v^{\prime}(t)+\frac{N-2}{2} v(t)=\frac{1}{2} R(t), \quad \text { for } t \in[0, \hat{t}]
$$

and

$$
t v^{\prime}(t)+\frac{N-2}{2} v(t)=-\frac{1}{2} R(t), \quad \text { for } t>\hat{t} .
$$

Lemma 2.2. Given $\hat{\lambda} \in(0, \infty)$, there exist $K_{1}, K_{2}$ and $\hat{d}(\hat{\lambda}):=\hat{d}$ such that if $\lambda<\hat{\lambda}$ and $d>\hat{d}$ then $K_{1} d^{-p / 2} \leq \hat{t} \leq K_{2} d^{-p / 2}$.

Proof. First we show that $K_{1}$ exists. Let $0<c<1$ be such that $v(\hat{t})=c d$. Since

$$
v^{\prime}(t)=t^{-N+1} \int_{0}^{t} s^{N-1}\left(-\lambda v(s)-|v(s)|^{p} v(s)\right) d s
$$

we have

$$
v^{\prime}(t) \geq-\frac{2 t}{N} d^{p+1}
$$

for $d$ sufficiently large, and all $t \in\left(0, t_{1}\right)$.

If $c<0.9$, then integrating $(2.10)$ over $[0, \hat{t}]$ we obtain

$$
\hat{t} \geq \sqrt{N(1-c)} d^{-p / 2} \geq \sqrt{\frac{N}{10}} d^{-p / 2} .
$$

On the other hand, from (2.9) we have

$$
v^{\prime}(t) \leq-\frac{t}{N} c^{p+1} d^{p+1}
$$

Thus, integrating $(2.12)$ on $[0, \hat{t}]$ we infer

$$
c d+\frac{\hat{t}^{2} c^{p+1} d^{p+1}}{N} \leq d .
$$

In particular, $\hat{t}^{2} c^{p+1} d^{p+1} / N \leq d$. Hence, if $c \geq .9$ then

$$
\hat{t}^{2} \leq \frac{d^{-p} N}{c^{p+1}} \leq \frac{d^{-p} N}{(.9)^{p+1}} \rightarrow 0
$$

as $d \rightarrow \infty$. From the definition of $\hat{t}$ and (2.4) we have

$$
(N-2)^{2}-\frac{8 \hat{t}^{2}}{p+2}|v(\hat{t})|^{p}-4 \lambda \hat{t}+\frac{8 \hat{t}^{2-N} \lambda}{v^{2}(\hat{t})} \int_{0}^{\hat{t}} r^{N-1} v^{2}(r) d r=0 .
$$

Using (2.14) we can assume that $4 \lambda \hat{t} \leq \frac{8 \hat{t}^{2}}{p+2}|v(\hat{t})|^{p}$, thus

$$
(N-2)^{2} \leq \frac{8 \hat{t}^{2}}{p+2}|v(\hat{t})|^{p}+4 \lambda \hat{t} \leq \frac{16 \hat{t}^{2}}{p+2} d^{p} .
$$


Hence

$$
\hat{t} \geq \sqrt{\frac{N(N-2)}{8}} d^{-p / 2} .
$$

Therefore from (2.11) and (2.17) we see that

$$
K_{1}=\min \left\{\sqrt{\frac{N(N-2)}{8}}, \sqrt{\frac{N}{10}}\right\} \text {. }
$$

Now we show that $K_{2}$ exists. Integrating $(2.10)$ over $\left[0, d^{-p / 2}\right]$ we obtain

$$
v(t) \geq d\left(1-\frac{1}{N}\right) \text {. }
$$

Suppose $\hat{t}>d^{-p / 2}$. For $t \in\left[d^{-p / 2}, \hat{t}\right]$ using (2.4) we see that

$$
\frac{t v^{\prime}(t)}{v(t)} \geq-\frac{N-2}{2}
$$

Integrating (2.20) for $t \in\left[d^{-p / 2}, \hat{t}\right]$ we infer

$$
\ln \left(\frac{v(t)}{v\left(d^{-p / 2}\right)}\right) \geq \ln \left(\frac{d^{-p / 2}}{t}\right)^{\frac{N-2}{2}}
$$

Hence

$$
v(t) \geq v\left(d^{-p / 2}\right) t^{-2 / p} d^{-1} \geq\left(1-\frac{1}{N}\right) t^{-2 / p}
$$

where we have also used (2.19).

From (2.6) and (2.21) we see that

$$
h(\hat{t}) \geq h\left(d^{-p / 2}\right)+\int_{d^{-p / 2}}^{\hat{t}} \frac{2}{N}\left(1-\frac{1}{N}\right)^{p} s^{-1} d s .
$$

Since $h$ is increasing and $h(\hat{t})=\frac{N-2}{2}$ we have

$$
\frac{N-2}{2} \geq \frac{2}{N}\left(1-\frac{1}{N}\right)^{p} \ln \left(\frac{\hat{t}}{d^{-p / 2}}\right) \text {. }
$$

Thus

$$
\hat{t} \leq \max \left\{1, e^{\frac{(N-2) N}{4}\left(1-\frac{1}{N}\right)^{-p}}\right\} d^{-p / 2}:=K_{2} d^{-p / 2}
$$

which together with $(2.18)$ proves the lemma. Now we define

$$
m(t, \lambda, d):=m(t)=v(t) t^{2 / p} .
$$

Corollary 2.3. (a) If $t \in(0, \hat{t})$ then $m^{\prime}(t)>0$, and if $t \in\left(\hat{t}, t_{1}\right)$ then $m^{\prime}(t)<0$. In particular, $m$ attains its maximum on $\left[0, t_{1}\right]$ at $\hat{t}$.

(b) For each $\lambda>0$

$$
\lim _{d \rightarrow \infty} m(\hat{t})=\left(\frac{N(N-2)}{4}\right)^{1 / p} .
$$


Proof. Since $m(t)=t^{2 / p} v(t)$, we have

$$
m^{\prime}(t)=\frac{2}{p} t^{2 / p-1} v(t)+t^{2 / p} v^{\prime}(t)=t^{2 / p-1}\left(\frac{N-2}{2} v(t)+t v^{\prime}(t)\right) .
$$

Thus (a) follows directly from (2.7)-(2.8). From (2.15) and the fact that $\hat{t} \rightarrow 0$ as $d \rightarrow \infty$ we see that $v(\hat{t}) \geq c d$ with $c$ independent of $d$. Therefore,

$$
\frac{8 \hat{t}^{2-N} \lambda}{v^{2}(\hat{t})} \int_{0}^{\hat{t}} r^{N-1} v^{2}(r) d r \rightarrow 0 \quad \text { as } d \rightarrow \infty
$$

This and (2.15) prove part (b). Hence, the corollary is proven.

Lemma 2.4. Let $\bar{t}>\hat{t}$ be such that $v(\bar{t})=\gamma \bar{t}^{-2 / p}$, where $\gamma=\left(\frac{N-2}{2}\right)^{2 / p}$. There exists $K_{3}>0$ such that $\bar{t} \leq K_{3} d^{-p / 2}$.

Proof. For $t \in[\hat{t}, \hat{t}]$ we have

$$
v(t) \geq \gamma t^{-2 / p}
$$

Hence, using (2.6) we infer

$$
h(t) \geq h(\hat{t})+\frac{2 \gamma^{p}}{N} \ln \left(\frac{t}{\hat{t}}\right) .
$$

Using the definition of $h(t)$ and the fact that $h(\hat{t})=\frac{N-2}{2}=\frac{2}{p}$ we see that

$$
-\frac{v^{\prime}(t)}{v(t)} \geq \frac{2}{p t}+\frac{2 \gamma^{p}}{N} \frac{1}{t} \ln \left(\frac{t}{\hat{t}}\right)
$$

Integrating (2.26) over $[\hat{t}, t]$ and using that $\hat{t}<1$ for $d$ large we obtain

$$
\ln \left(\frac{v(\hat{t})}{v(t)}\right) \geq \ln \left(\frac{t}{\hat{t}}\right)^{2 / p}+\frac{\gamma^{p}}{N}(\ln t-\ln \hat{t})^{2}
$$

Hence

$$
\frac{v(\hat{t})}{v(t)} \geq\left(\frac{t}{\hat{t}}\right)^{2 / p} e^{\left(\gamma^{p} / N\right)(\ln (t / \hat{t}))^{2}}
$$

From (2.25) and (2.27) and the definition of $m(t)$ (see (2.24)) we have

$$
\gamma \leq v(\bar{t}) \bar{t}^{2 / p} \leq m(\hat{t}) e^{-\left(\gamma^{p} / N\right)(\ln (t / \hat{t}))^{2}}
$$

Now, from $(2.28)$ we see that $e^{\left(\gamma^{p} / N\right)(\ln (t / \hat{t}))^{2}} \leq m(\hat{t}) / \gamma$. Hence

$$
\ln \left(\frac{t}{\hat{t}}\right) \leq\left(\frac{N}{\gamma^{p}} \ln \left(\frac{m(\hat{t})}{\gamma}\right)\right)^{1 / 2}:=K_{3}^{\prime} .
$$

Therefore, from (2.29) and Lemma 2.2 we obtain

$$
t \leq \hat{t} e^{K_{3}^{\prime}} \leq K_{3} d^{-p / 2}
$$

for every $t \in[\hat{t}, \hat{t}]$, which concludes the proof of the lemma.

Next, we estimate the decay of $m(t)$. 
Lemma 2.5. Let $A \in(0,(N-2) / \sqrt{2 N})$. Given $\bar{\lambda}>0$ there exists $d_{0}:=d_{0}(\bar{\lambda})$ such that

$$
m(t):=m(t, \lambda, d) \leq m(\bar{t})\left(\frac{\bar{t}}{t}\right)^{A},
$$

for $t \in\left[\bar{t}, s_{1}:=\left((N-2)^{2} / 2 N-A^{2}\right) / \bar{\lambda}\right], \lambda \in(0, \bar{\lambda})$ and $d>d_{0}$.

Proof. Let $R(t)$ be as in (2.4). Since $\bar{t} \leq t \leq s_{1}$, we have that $R(t) \geq 2 A v(t)$. From (2.8) using the definition of $m(t)$ we obtain

$$
m^{\prime}(t)=-\frac{1}{2} t^{\frac{2}{p}-1} R(t) \leq-A \frac{m}{t} .
$$

Integrating (2.30) on $[\bar{t}, t]$ and using the fact that $m$ is decreasing we have

$$
m(t) \leq m(\bar{t})\left(\frac{\bar{t}}{t}\right)^{A}
$$

which proves the lemma.

Corollary 2.6. If $N=3,4,5$, then $v(\cdot, \lambda, d)$ converges uniformly to zero in compact subset of $(0, \infty)$ when $d \rightarrow \infty$.

Proof. For $N=4,5$ see [1]. If $N=3$ and $d$ sufficiently large from [1] we know that $v(\cdot, \lambda, d)>0$ on $[0,1 / \sqrt{\lambda}]$. Thus for $1>T>0$ given we have

$$
v(T) \leq m(\bar{t})(\bar{t})^{A}(T)^{-A-1 / 2} .
$$

Hence, $v(T)$ converges to zero as $d \rightarrow \infty$. Also, from (2.4) it follows that $v^{\prime}(T) \rightarrow 0$ as $d \rightarrow \infty$. Since

$$
\frac{d}{d t}\left(\left(v^{\prime}(t)\right)^{2}+\lambda v^{2}(t)+\frac{|v(t)|^{p+2}}{p+2}\right) \leq 0
$$

we see that $v \rightarrow 0$ on $[T, \infty)$ as $d \rightarrow \infty$, which proves the corollary.

3. ANALYSIS OF $v_{\lambda}$ AND $v_{d}$

Let $v_{\lambda}$ and $v_{d}$ denote derivatives of $v$ with respect to $\lambda$ and $d$. Defining, for $\rho>0$ and $d>0$,

$$
\omega(t)=v(\rho t, \lambda, d)
$$

we see that

$$
\begin{gathered}
\omega^{\prime \prime}(t)+\frac{N-1}{t} \omega^{\prime}(t)+\rho^{2} \lambda \omega(t)+\rho^{2}|\omega(t)|^{p} \omega(t)=0, \\
\omega(0)=d, \quad \omega^{\prime}(0)=0 .
\end{gathered}
$$

Multiplying by $\rho^{2 / p}$ and letting $\xi(t):=\rho^{2 / p} \omega(t)$ we infer

$$
\begin{gathered}
\xi^{\prime \prime}(t)+\frac{N-1}{t} \xi^{\prime}(t)+\rho^{2} \lambda \xi(t)+|\xi(t)|^{p} \xi(t)=0, \\
\xi(0)=\rho^{2 / p} d, \quad \xi^{\prime}(0)=0 .
\end{gathered}
$$

Hence

$$
\xi(t)=v\left(t, \rho^{2} \lambda, \rho^{2 / p} d\right) .
$$


Thus, from the definition of $\xi, \omega$ and (3.4) we obtain

$$
\rho^{2 / p} v(\rho t, \lambda, d)=v\left(t, \lambda \rho^{2}, \rho^{2 / p} d\right) .
$$

Differentiating (3.5) with respect to $\rho$ we see that

$$
\begin{aligned}
& \frac{2}{p} \rho^{2 / p-1} v(\rho t, \lambda, d)+\rho^{2 / p} t v^{\prime}(\rho t, \lambda, d) \\
& \quad=-2 \lambda \rho v_{\lambda}\left(t, \lambda \rho^{2}, \rho^{2 / p} d\right)+\frac{2}{p} d \rho^{2 / p-1} v_{d}\left(t, \lambda \rho^{2}, \rho^{2 / p}\right) .
\end{aligned}
$$

Hence, for $\rho=1$ we have

$$
2 \lambda v_{\lambda}(t, \lambda, d)+\frac{2}{p} d v_{d}(t, \lambda, d)=t v^{\prime}(t, \lambda, d)+\frac{N-2}{2} v(t, \lambda, d) .
$$

Throughout the rest of the paper we assume $\lambda$ to be in a bounded set. Now, we analyze some properties of $v_{\lambda}$ and $v_{d}$.

Lemma 3.1. For $t \in\left[0, t_{1}\right]$ we have

$$
\left(v_{\lambda}^{\prime}(t)\right)^{2}+\lambda\left(v_{\lambda}(t)\right)^{2}+(p+1)|v(t)|^{p}\left(v_{\lambda}(t)\right)^{2} \leq\left(\int_{0}^{t} v(s) d s\right)^{2},
$$

where $t_{1}$ is the first zero of $v$.

Proof. We define

$$
E_{1}(t)=\frac{\left(v_{\lambda}^{\prime}(t)\right)^{2}}{2}+\frac{\lambda\left(v_{\lambda}(t)\right)^{2}}{2}+\frac{(p+1)|v(t)|^{p}\left(v_{\lambda}(t)\right)^{2}}{2} .
$$

Hence

$$
\begin{aligned}
E_{1}^{\prime}(t)= & v_{\lambda}^{\prime}(t) v_{\lambda}^{\prime \prime}(t)+\lambda v_{\lambda}(t) v_{\lambda}^{\prime}+\frac{(p+1) p|v(t)|^{p-1}\left(v_{\lambda}(t)\right)^{2} v^{\prime}(t)}{2} \\
& +(p+1)|v(t)|^{p} v_{\lambda}(t) v_{\lambda}^{\prime}(t) \\
= & -\frac{N-1}{t}\left(v_{\lambda}^{\prime}(t)\right)^{2}-v(t) v_{\lambda}^{\prime}(t)+\frac{(p+1) p|v(t)|^{p-1} v^{\prime}(t)\left(v_{\lambda}(t)\right)^{2}}{2} \\
\leq & v(t)\left|v_{\lambda}^{\prime}(t)\right| \leq v(t) \sqrt{2 E_{1}(t) .}
\end{aligned}
$$

Thus

$$
\frac{E_{1}^{\prime}(t)}{\sqrt{2 E_{1}(t)}} \leq v(t)
$$

Hence, integrating on $[0, t]$ the lemma follows. Let $\xi_{1} \in\left(0, \gamma^{p}\right)$ be such that if $\xi \in\left(0, \xi_{1}\right)$, then

$$
g(\xi)=\frac{\frac{(N-2)^{2}}{4}+\frac{N-2}{4} \sqrt{(N-2)^{2}-\frac{8 \xi}{p+2}}-\xi}{\frac{1}{2} \sqrt{(N-2)^{2}-\frac{8 \xi}{p+2}}}<\frac{N-2}{2} .
$$

Such a $\xi_{1}$ exists because $g(0)=\frac{N-2}{2}$ and $g^{\prime}(0)<0$. Let

$$
\xi \in\left(0, \min \left\{\xi_{1}, m(\hat{t})\right\}\right) .
$$


Since $m(t)$ is a continuous function there exists $s_{2} \in\left(0, t_{1}\right)$ such that

$$
\left|v\left(s_{2}\right)\right|^{p}=\frac{\xi}{p+1} s_{2}^{-2} .
$$

Note that by Lemma 2.5 for $d$ sufficiently large we have

$$
s_{2} \leq \xi^{-1 / A}((p+1) m(\bar{t}))^{1 / A} \bar{t} .
$$

This and Lemma 2.4 show that

$$
4 \lambda s_{2}+\frac{8 s_{2}^{2-N} \lambda}{v^{2}(t)} \int_{0}^{s_{2}} r^{N-1} v^{2}(r) d r \rightarrow 0 \quad \text { as } d \rightarrow \infty .
$$

Let $\mu(\xi):=\mu>0$ be such that

$$
\frac{\mu+\frac{(N-2)^{2}}{4}+\frac{N-2}{4} \sqrt{(N-2)^{2}-\frac{8 \xi}{p+2}+\mu}-\xi}{\frac{1}{2} \sqrt{(N-2)^{2}-\frac{8 \xi}{p+2}-\mu}-\mu}<\frac{N-2}{2} .
$$

By Lemma 3.1 and (3.11) there exists $d_{2}$ such that for $d>d_{2}(\xi)$

(3.13) $\left|\frac{2 s_{2} \lambda v_{\lambda}^{\prime}\left(s_{2}\right)}{v\left(s_{2}\right)}\right|+\left|\frac{2 \lambda v_{\lambda}\left(s_{2}\right)}{v\left(s_{2}\right)}\right|+4 \lambda s_{2}+\left|\frac{8 s_{2}^{2-N} \lambda}{v^{2}(t)} \int_{0}^{s_{2}} r^{N-1} v^{2}(r) d r\right|<\mu$.

Now, we prove the following lemma.

Lemma 3.2. If $\xi$ and $s_{2}$ are as above, then

$$
\left|\frac{s_{2} v_{d}^{\prime}\left(s_{2}\right)}{v_{d}\left(s_{2}\right)}\right|<\frac{N-2}{2} \text {. }
$$

Proof. Differentiating (3.6) with respect to $t$ and using (1.2) and (2.4) we obtain

$$
\begin{aligned}
2 \lambda v_{\lambda}^{\prime}(t) & +\frac{2 d}{p} v_{d}^{\prime}(t)=\frac{N-2}{2} v^{\prime}(t)+t v^{\prime \prime} \\
= & \frac{2-N}{2} v^{\prime}(t)-\lambda v(t) t-t|v(t)|^{p} v(t) \\
= & \frac{(N-2)^{2}}{4 t} v(t)+\frac{N-2}{4 t} R(t)-\lambda v(t) t-t|v(t)|^{p} v(t) .
\end{aligned}
$$

Hence

$$
\begin{aligned}
\left|\frac{s_{2} v_{d}^{\prime}\left(s_{2}\right)}{v_{d}\left(s_{2}\right)}\right| & =\left|\frac{2 s_{2} \lambda v_{\lambda}^{\prime}\left(s_{2}\right)-\frac{(N-2)^{2}}{4} v\left(s_{2}\right)-\frac{N-2}{4} R\left(s_{2}\right)+\lambda s_{2}^{2} v\left(s_{2}\right)+s_{2}^{2}\left|v\left(s_{2}\right)\right|^{p} v\left(s_{2}\right)}{-2 \lambda v_{\lambda}-\frac{1}{2} R\left(s_{2}\right)}\right| \\
& \leq \frac{\mu+\frac{(N-2)^{2}}{4}+\frac{N-2}{4} \sqrt{(N-2)^{2}-\frac{8 \xi}{p+2}+\mu}-\xi}{-\left|\frac{2 \lambda v_{2}\left(s_{2}\right)}{v\left(s_{2}\right)}\right|+\frac{1}{2} \sqrt{(N-2)^{2}-\frac{8 \xi}{p+2}-\mu}}<\frac{N-2}{2} .
\end{aligned}
$$

where we have used (3.12) and (3.13). Thus, the lemma is proven. 
Now we show that for $d$ large

$$
v_{d}(t)<0, \quad v_{d}^{\prime}(t)>0
$$

for all $t \in\left(\bar{t}, s_{2}\right)$. In fact, from (2.8) and (3.6) we have

$$
\begin{aligned}
\frac{2 d}{p} v_{d}(t) & =-2 \lambda v_{\lambda}(t)-\frac{1}{2} R(t) \\
& \leq-2 \lambda v_{\lambda}(t)-\frac{1}{2} \sqrt{\left[(N-2)^{2}-4 t \lambda-\frac{8 t^{2} \lambda \gamma}{p+2}\right] m^{2}(t)(t)^{-4 / p}} \\
& \leq k d^{1-p}-\bar{k} m\left(s_{2}\right) d<0,
\end{aligned}
$$

where we have also used Lemma 3.1. Hence $v_{d}(t)<0$.

On the other hand, from (3.14) and Lemma 3.1 we have

$$
\frac{2 d}{p} v_{d}^{\prime}(\bar{t}) \geq-k d^{1-p / 2}+\frac{v(\bar{t})}{\bar{t}}\left\{\frac{(N-2)^{2}}{4}-(\bar{t})^{2}\left[\lambda+|v(\bar{t})|^{p}\right]\right\} .
$$

Since $|m(\bar{t})|^{p}<\frac{(N-2)^{2}}{4}$ for $d$ sufficiently large from (3.18) we obtain

$$
\frac{2 d}{p} v_{d}^{\prime}(\bar{t}) \geq-k d^{1-p / 2}+\bar{k} d^{1+p / 2}>0 .
$$

Thus, for $t \in\left(\bar{t}, s_{2}\right)$ we have

$$
t^{N-1} v_{d}^{\prime}(t)=-\int_{\bar{t}}^{t} r^{N-1}\left(\lambda+(p+1)|v(r)|^{p}\right) v_{d}(r) d r+(\bar{t})^{N-1} v_{d}^{\prime}(\bar{t})
$$

Therefore, using (3.17), (3.19), and (3.20) we see that $v_{d}^{\prime}(t)>0$ which proves (3.16).

\section{Oscillations oF $v_{d}$ FOR $d$ LARGE}

Let $t_{1}<t_{2}<\cdots<t_{j}=1$ be the zeroes of $v(\cdot, \lambda, d)$ in $(0,1]$. Let $\bar{t}$ be as in Lemma 2.4. By (3.16) we know that $v_{d}(\bar{t})<0$. Let $\tau_{1}<\tau_{2}<\cdots<\tau_{i}<\cdots$ be the zeroes of $v_{d}(\cdot, \lambda, d)$ on $(\bar{t}, 1]$. In this section (see Lemma 4.2 below) we show that the zeroes of $v$ separate the zeroes of $v_{d}$. In the proof of our next lemma we compare the equation

$$
v_{d}^{\prime \prime}(t)+\frac{N-1}{t} v_{d}^{\prime}(t)+\left(\lambda+(p+1)|v(t)|^{p}\right) v_{d}(t)=0
$$

with the Cauchy-Euler equation

$$
z^{\prime \prime}(t)+\frac{N-1}{t} z^{\prime}(t)+\frac{(N-2)^{2}}{4 t^{2}} z(t)=0
$$

Since $z(r)=r^{-\frac{N-2}{2}}$ is a solution to (4.2) and $z(r) \neq 0$; by the Sturm comparison theorem if there exist $r_{0}$ and $r_{1}$, such that $r_{0}<r_{1},\left|\frac{v_{d}^{\prime}\left(r_{0}\right)}{v_{d}\left(r_{0}\right)}\right|<\left|\frac{z^{\prime}\left(r_{0}\right)}{z\left(r_{0}\right)}\right|$ and $\lambda+(p+1)|v(r)|^{p} \leq \frac{(N-2)^{2}}{r^{2}}$ on $\left(r_{0}, r_{1}\right)$, then $v_{d}$ cannot have zeroes in $\left(r_{0}, r_{1}\right)$ (see [8]). More precisely $\left|\frac{v_{d}^{\prime}(t)}{v_{d}(t)}\right|<\left|\frac{z^{\prime}(t)}{z(t)}\right|$ for all $t \in\left[r_{0}, r_{1}\right]$. Now we are ready to estimate $\tau_{1}$. 
Lemma 4.1. For $d$ sufficiently large

$$
\tau_{1} \geq \frac{0.49(N-2)}{\sqrt{\lambda}} .
$$

Moreover, for $N=3$,

$$
\tau_{1}>\frac{1.7}{\sqrt{\lambda}}
$$

Proof. Let $\xi_{1}$ and $s_{2}$ be as in (3.9)-(3.10), and let $\xi \in\left(0, \min \left\{\xi_{1}, m(\hat{t})\right)\right.$ be such that

$$
\sqrt{(N-2)^{2}-4(p+1) \xi} \geq .99(N-2) .
$$

Since $v(t) \leq m\left(s_{2}\right) t^{-2 / p}$ for $t \in\left(s_{2}, t_{1}\right)$ and $v$ converges to zero on $\left(t_{1}, \infty\right)$ as $d \rightarrow \infty$, we see that for $d$ sufficiently large

$$
\lambda+(p+1)|v(t)|^{p} \leq\left(\frac{N-2}{2}\right)^{2} t^{-2}
$$

if $t \in\left[s_{2}, s_{3}:=\frac{1}{\sqrt{\lambda}} \frac{\sqrt{(N-2)^{2}-4(p+1) \xi}}{2}\right]$. Thus, by Lemma 3.2 and the Sturm comparison theorem (see (4.1)-(4.2)) we have

$$
\left|\frac{v_{d}^{\prime}(s)}{v_{d}(s)}\right| \leq\left|\frac{z^{\prime}(s)}{z(s)}\right|=\frac{N-2}{2 s}
$$

for all $s \in\left[s_{2}, s_{3}\right]$. Hence $v_{d}<0$ on $\left[s_{2}, s_{3}\right]$. Therefore, (4.3) follows from (4.5) and (4.7).

Now, we consider the case $N=3$. Let, $\psi$ be the argument function such that

$$
\begin{aligned}
& v_{d}(t)=-r(t) \cos \psi(t), \quad v_{d}^{\prime}(t)=r(t) \sin \psi(t), \\
& r^{2}(t)=v_{d}^{2}(t)+\left(v_{d}^{\prime}(t)\right)^{2}, \quad \psi\left(\tau_{0}\right)=0,
\end{aligned}
$$

where $\tau_{0}=\inf \left\{t<\bar{t} ; v_{d}^{\prime}(t) \geq 0\right\}$. Because $v_{d}^{\prime}(t)<0$ in a neighborhood of 0 we see that $\tau_{0}>0$. From (4.8) we have

$$
\psi^{\prime}(t)=\frac{1+\frac{2}{t}(-\tan \psi(t))+5|v(t)|^{4}}{1+\tan ^{2} \psi(t)} .
$$

Let $s_{4}:=\inf \left\{s>s_{3} ; \tan (s) \geq 1.02 \sqrt{\lambda}\right\}$. From the definition of $s_{3}$ and (4.5) we have that $\tan s_{3} \leq \frac{N-2}{2 s_{3}} \leq \frac{\sqrt{\lambda}}{.99}<1.02 \sqrt{\lambda}$. Hence $\psi^{\prime}\left(s_{4}\right) \geq 0$. Thus, from (4.9) we infer

$$
1+\frac{-\frac{2}{s_{4}} 1.02 \sqrt{\lambda}+5\left|v\left(s_{4}\right)\right|^{4}+\lambda-1}{1+(1.02)^{2} \lambda} \geq 0 .
$$

Since $v$ converges to zero on compact subsets of $(0, \infty)$ we have

$$
s_{4} \geq \frac{2.04 \sqrt{\lambda}}{\lambda+(1.02)^{2} \lambda+5\left|v\left(s_{4}\right)\right|^{4}} \geq \frac{.99}{\sqrt{\lambda}} .
$$


Let now

$$
Y(t)=v_{d}\left(\frac{t}{\sqrt{\lambda}}\right)
$$

and let $\alpha$ be such that

$$
\begin{aligned}
& Y(t)=-\sqrt{Y^{2}(t)+\left(Y^{\prime}(t)\right)^{2}} \cos \alpha(t), \\
& Y^{\prime}(t)=\sqrt{Y^{2}(t)+\left(Y^{\prime}(t)\right)^{2}} \sin \alpha(t) .
\end{aligned}
$$

It can easily be shown that $Y$ satisfies the equation

$$
Y^{\prime \prime}(t)+\frac{5}{t} Y^{\prime}(t)+\left(1+5 \frac{|v(t / \sqrt{\lambda})|^{4}}{\lambda}\right) Y(t)=0
$$

and that

$$
\alpha^{\prime}(t)=1+\frac{\frac{5}{t}(-\tan \alpha(t))+\frac{5}{\lambda}|v(t)|^{4}}{1+\tan ^{2} \alpha(t)} .
$$

Since $Y\left(s_{4} \sqrt{\lambda}\right)=v_{d}\left(s_{4}\right)$ and $Y^{\prime}\left(s_{4}(\sqrt{\lambda})=\frac{1}{\sqrt{\lambda}} v_{d}^{\prime}\left(s_{4}\right)\right.$, we have

$$
\left|\frac{Y^{\prime}\left(s_{4} \sqrt{\lambda}\right)}{Y\left(s_{4} \sqrt{\lambda}\right.}\right|=\frac{1}{\sqrt{\lambda}}\left|\frac{v_{d}^{\prime}\left(s_{4}\right)}{v_{d}\left(s_{4}\right)}\right|=1.02
$$

Because $\alpha\left(s_{4} \sqrt{\lambda}\right)=\arctan 1.02 \leq 0.796$ and $\alpha^{\prime}(t) \leq 1+\frac{5}{\lambda}\left|v\left(s_{4}\right)\right|^{4}($ see $(4.10)$ and (4.12)) we obtain

$$
\alpha(1.7) \leq .796+\left(1+\frac{5}{\lambda}\left|v\left(s_{4}\right)\right|^{4}\right)(1.7-.99) \leq .796+.72 \leq 1.512<\frac{\pi}{2} .
$$

Hence $Y<0$ on $\left(s_{4} \sqrt{\lambda}, 1.7\right)$. Thus $v_{d}<0$ on $\left[s_{4}, \frac{1.7}{\sqrt{\lambda}}\right]$ which proves (4.4), and therefore concludes the proof of the lemma.

Let $\tau_{0}$ be as in (4.8).

Lemma 4.2. For $d$ sufficiently large the zeros of $v$ separate the zeroes of $v_{d}$ on $\left[\tau_{0}, 1\right]$. Moreover, $v^{\prime}(1, \lambda, d) \cdot v_{d}(1, \lambda, d)>0$.

Proof. Let $T_{1}$ be the zero of $v^{\prime}$ on $\left[t_{1}, t_{2}\right]$. Suppose $\tau_{1}<T_{1}$. We show inductively that $\tau_{i} \in\left(t_{i}, t_{i+1}\right)$. From [1] we know that $t_{1}(\lambda, d) \rightarrow 0$ as $d \rightarrow \infty$ for $N=4,5$ and that $t_{1}(\lambda, d) \rightarrow \frac{\pi}{2 \sqrt{\lambda}}$ as $d \rightarrow \infty$ for $N=3$ (see [1]). This and Lemma 4.1 show that $\tau_{1}>t_{1}$ for $d$ large. By the Sturm comparison theorem it follows that $\tau_{1}<t_{2}$. Hence $\tau_{1} \in\left(t_{1}, t_{2}\right)$.

Suppose $\tau_{i} \in\left(t_{i}, t_{i+1}\right)$. Now we show that $\tau_{i+1} \in\left(t_{i+1}, t_{i+2}\right)$. Suppose $\tau_{i+1}<t_{i+1}$. Multiplying (1.2) by $r^{N-1} v_{d}(r)$ and (4.1) by $r^{N-1} v(r)$, integrating by parts on $\left[\tau_{1}, \tau_{i+1}\right]$ and subtracting we obtain

$$
\begin{array}{r}
\tau_{i+1}^{N-1} v_{d}^{\prime}\left(\tau_{i+1}\right) v\left(\tau_{i+1}\right)-\tau_{1}^{N-1} v_{d}^{\prime}\left(\tau_{1}\right) v\left(\tau_{1}\right) \\
=-p \int_{\tau_{1}}^{\tau_{i+1}} r^{N-1}|v(r)|^{p} v_{d}(r) v(r) d r .
\end{array}
$$


Without loss of generality we can assume $i$ to be even, thus $v_{d}^{\prime}\left(\tau_{i+1}\right)>0$ and $v>0$ on $\left[t_{i}, t_{i+1}\right]$. Also, since

$$
\begin{aligned}
\left(\left(v_{d}^{\prime}(t)\right)^{2}+\left(\lambda v_{d}(t)\right)^{2}\right)^{\prime} & \leq-2(p+1)|v(t)|^{p} v_{d}(t) v_{d}^{\prime}(t) \\
& \leq \frac{(p+1)|v(t)|^{p}}{\sqrt{\lambda}}\left(\left(v_{d}^{\prime}(t)\right)^{2}+\left(\lambda v_{d}(t)\right)^{2}\right)
\end{aligned}
$$

and $|v|$ converges to zero on $\left[t_{1}, 1\right]$ (see Corollary 2.6), we infer

$$
\left|v_{d}(t)\right| \leq \frac{\sqrt{e}}{\sqrt{\lambda}}\left|v_{d}^{\prime}\left(\tau_{1}\right)\right|
$$

On the other hand since $v$ is convex on $\left(t_{1}, T_{1}\right)$ we have

$$
v\left(\tau_{1}\right) \leq \frac{v\left(T_{1}\right)}{T_{1}-t_{1}}\left(\tau_{1}-t_{i}\right)
$$

Therefore (see Lemma 4.1)

$$
\left|v\left(\tau_{1}\right)\right| \geq \frac{\left|v\left(T_{1}\right)\right|}{T_{1}-t_{i}}\left|\tau_{1}-t_{1}\right| \geq k\left|v\left(T_{1}\right)\right| .
$$

Thus, from (4.15)-(4.17) we have

$$
\begin{aligned}
\left|\tau_{1}^{N-1} v\left(\tau_{1}\right) v_{d}^{\prime}\left(\tau_{1}\right)\right| & \leq \int_{\tau_{1}}^{\tau_{i+1}} r^{N-1} p|v(r)|^{p}|v(r)|\left|v_{d}(r)\right| d r \\
& \leq \frac{\sqrt{e}}{\sqrt{\lambda}} p\left|v\left(T_{1}\right)\right|^{p+1}\left|v_{d}^{\prime}\left(\tau_{1}\right)\right| .
\end{aligned}
$$

Hence

$$
\left|v\left(\tau_{1}\right)\right| \leq \frac{p \sqrt{e}}{\sqrt{\lambda}}\left|v\left(T_{1}\right)\right|^{p+1} .
$$

Combining (4.17) and (4.18) we see that there exists $M>0$ such that $\left|v\left(T_{1}\right)\right|^{p}$ $\geq M$ which is a contradiction since $v \rightarrow 0$ as $d \rightarrow \infty$. Hence $\tau_{i+1}>t_{i+1}$.

On the other hand, if $\tau_{1} \in\left[T_{1}, t_{2}\right]$ we let $\omega$ be the solution to

$$
\begin{gathered}
\omega^{\prime \prime}(r)+\frac{N-1}{r} \omega^{\prime}(r)+\left(\lambda+(p+1)|v(r)|^{p}\right) \omega(r)=0, \\
\omega\left(T_{1}\right)=0, \quad \omega^{\prime}\left(T_{1}\right)=1 .
\end{gathered}
$$

Let $T_{1}=\sigma_{1}<\sigma_{2}<\cdots<\sigma_{n}<\cdots$ be the zeroes of $\omega$. We claim that

$$
\sigma_{1}<t_{2}<\sigma_{2}<\cdots<t_{i}<\sigma_{i}<t_{i+1}<\cdots<\sigma_{j-1}<1=t_{j}<\sigma_{j} .
$$

In fact, cross-multiplying equations (4.19) and (1.2), integrating by parts on $\left[\tau_{1}, \tau_{j+1}\right]$ and arguing as above, we see that the zeroes of $v$ separate the zeroes of $\omega$, hence (4.21) holds. Now, since $\omega$ and $v_{d}$ satisfy the same linear equation by the Sturm comparison theorem we know that the zeroes of $\omega$ separate the zeroes of $v_{d}$ and conversely. Hence we have

$$
\cdots<t_{i}<\sigma_{i}<\tau_{i}<t_{i+1}<\cdots,
$$

which proves that the zeroes of $v$ separate the zeroes of $v_{d}$. 
From the above discussion it follows that because $v_{d}^{\prime}\left(\tau_{1}\right)>0$, we have $\operatorname{sgn} v_{d}^{\prime}\left(\tau_{i}\right)=(-1)^{i+1}$ and because $v\left(\tau_{1}\right)<0$, we have $\operatorname{sgn} v\left(\tau_{i}\right)=(-1)^{i}$, hence $v_{d}^{\prime}\left(\tau_{i}\right) v\left(\tau_{i}\right)<0$. Finally, if $v^{\prime}(1)>0$, then $v<0$ on $\left(t_{j-1}, t_{j}=1\right)$. Thus, $v_{d}^{\prime}\left(\tau_{j-1}\right)>0$. Hence, $v_{d}(1)>0$. Similarly, if $v^{\prime}(1)<0$ then $v_{d}(1)<0$, which concludes the proof of the lemma.

\section{BifURCATION ANALYSIS}

In this section we summarize qualitative properties of the solution set to equation (1.1). We make extensive use of the results in [1] as well as of the properties of the solution to

$$
\begin{gathered}
y^{\prime \prime}(r)+\frac{N-1}{r} y^{\prime}(r)+\lambda\left(y(r)+|y(r)|^{p} y(r)\right)=0, \\
y(0)=d, \quad y^{\prime}(0)=0, \\
y(1)=0 .
\end{gathered}
$$

We will denote by $y(r, \lambda, d)$ the solution to the initial value problem (5.1)(5.2). In order to state the following lemma we denote by $\mu_{1}<\mu_{2}<\ldots$ the eigenvalues of $-\Delta$ restricted to the space of radial functions with a zero Dirichlet boundary condition on $B$.

Lemma 5.1. (a) $G \subset(0, \infty) \times(0, \infty)$ is a connected component of $\{(\lambda, d)$; $y(1, \lambda, d)=0\}$ if and only if there exists a differentiable function $\beta:(0, \infty) \rightarrow$ $(0, \infty)$, and a positive integer $k$ such that $G=\{(\beta(d), d) ; d>0\}$, and $y(\cdot, \beta(d), d)$ has $k$ zeroes in $(0,1]$ for each $d>0$. In addition, we have:

(i) If $N=3$ then $\beta(d) \rightarrow(k \pi)^{2}$ as $d \rightarrow 0$ and $\beta(d) \rightarrow(k-(1 / 2))^{2} \pi^{2}$ as $d \rightarrow \infty$.

(ii) If $N=4$, 5 then $\beta(d) \rightarrow \mu_{k}$ as $d \rightarrow 0$, and $\beta(d) \rightarrow \mu_{k-1}$ as $d \rightarrow \infty$, where $\mu_{0}=0$.

(b) Conversely, for each positive integer $k$ there exists a unique differentiable function $\beta_{k}:=\beta:(0, \infty) \rightarrow(0, \infty)$ such that $y(\cdot, \beta(d), d)$ is a solution to (5.1)-(5.3), $y(\cdot, \beta(d), d)$ had $k$ zeroes in $(0,1]$, and satisfies (i) and (ii).

Proof. (a) From the definition of $y$ we have

$$
y(\rho r, \lambda, d)=y\left(r, \lambda \rho^{2}, d\right) .
$$

Therefore, differentiating with respect to $\rho$ and replacing $\rho$ by 1 , we have

$$
y^{\prime}(1, \lambda, d)=2 \lambda y_{\lambda}(r, \lambda, d) .
$$

Since, by uniqueness of solutions to the initial value problem $(5.1)-(5.2)$, we know that $y(\cdot, \lambda, d)$ cannot have degenerate zeroes we see that if $y(r, \lambda, d)=$ 0 then $y^{\prime}(r, \lambda, d) \neq 0$. Thus, by the implicit function theorem, if $G$ $\subset(0, \infty) \times(0, \infty)$ is a connected component of $\{(\lambda, d) ; \lambda>0, d>0$, $y(1, \lambda, d)=0\}$, then $G=\{(\beta(d), d) ; d \in(a, b)\}$, with $\beta$ continuous. 
Again, by the nondegeneracy of the zeroes of $y(\cdot, \lambda, d)$ we have that the number of zeroes of $v(\cdot, \beta(d), d)$ is a constant $k$. Hence, by the Sturm comparison theorem, $\beta(d) \leq \mu_{k}$ for all $d \in(a, b)$. Suppose $b<\infty$. Let $\left\{d_{n}\right\}$ be a sequence converging to $b$. Since $\beta$ is bounded, without loss of generality we can assume that $\left\{\beta\left(d_{n}\right)\right\}$ converges, say to $c$. By the continuity of $y$ we see that $y(1, c, b)=0$. Since $b \neq 0$ we know that $c \neq 0$. Thus $(c, b) \in G$, which contradicts that $G$ is a connected component. This contradiction shows that $b=+\infty$. A similar argument shows that $a=0$. Thus $G=\{(\beta(d), d) ; d>0\}$. Since, by standard bifurcation arguments, the only solutions to $(5.1)-(5.3)$ having $k$ zeroes in $(0,1]$ are those bifurcating from $\left(\mu_{k}, 0\right)$ we see that $\beta(d) \rightarrow \mu_{k}$ as $d \rightarrow 0$. Also from [1], it follows that the only solutions to (5.1)-(5.3) with $d$ large and $k$ zeroes in $(0,1]$ with $d$ near 0 , are those bifurcating from $\left(\mu_{k-1}, \infty\right)$ if $N=4,5((0, \infty)$ if $k=1)$ or $\left(\left(k-\left(\frac{1}{2}\right)\right)^{2} \pi^{2}, \infty\right)$ if $N=3$. Thus $\beta$ satisfies (i), (ii). This proves part (a).

(b) By the local bifurcation theory for simple eigenvalues (see [7]), for each $k=1,2, \ldots$ there exists $\nu>0$ and a continuous function $\beta_{k}:(0, \nu) \rightarrow$ $(0, \infty)$ such that $y\left(\cdot, \beta_{k}(d), d\right)$ is a solution to $(5.1)-(5.3)$. Moreover, $y\left(\cdot, \beta_{k}(d), d\right)$ has $k$ zeroes in $(0,1]$. Letting $G$ denote the connected component of $\{(\lambda, d) ; \lambda>0, d>0, y(1, \lambda, d)=0\}$ containing $\left\{\left(\beta_{k}(d), d\right) ; d \in\right.$ $(0, \nu)\}$, by part (a) we see that $\beta_{k}$ can be extended to $(0, \infty)$. Since the zeroes of solutions to $(5.1)-(5.3)$ are nondegenerate we see that each $y\left(\cdot, \beta_{k}(d), d\right)$ has $k$ zeroes in $(0,1]$.

In order to prove uniqueness we let $\beta_{k}$ and $\beta_{k}^{\prime}$ be continuous functions such that $v\left(1, \beta_{k}(d), d\right)=0, v\left(1, \beta_{k}^{\prime}(d), d\right)=0$, and $v\left(\cdot, \beta_{k}(d), d\right)$, as well as $v\left(\cdot, \beta_{k}^{\prime}(d), d\right)$ has $k$ zeroes. Then $\beta_{k}(d) \rightarrow \mu_{k}$ as $d \rightarrow 0$ and $\beta_{k}^{\prime}(d) \rightarrow$ $\mu_{k}$ as $d \rightarrow 0$. Since $\mu_{k}$ is a simple eigenvalue, for $d$ small enough we see that $\beta_{k}(d)=\beta_{k}^{\prime}(d)$. Furthermore, because $\left\{\left(\beta_{k}(d), d\right) ; d \in(0, \infty)\right\}$ and $\left\{\left(\beta_{k}^{\prime}(d), d\right) ; d \in(0, \infty)\right\}$ are connected components of solutions then they are equal, which proves that $\beta_{k}=\beta_{k}^{\prime}$. Hence, the lemma is proven.

From the definition of $y$ and $v$ we have

$$
\lambda^{-1 / p} v\left(r, \lambda, d \lambda^{1 / p}\right)=y(r, \lambda, d) .
$$

This relation allows us to transfer the bifurcation properties to $y$ to $v$. Indeed we have

Lemma 5.2. (a) $\Gamma$ is a connected component of $\{(\lambda, d) ; v(1, \lambda, d)=0\}$ if and only if there exists a positive integer $k$ such that $\Gamma=\left\{\left(\beta_{k}(d), \beta_{k}(d)^{1 / p} d\right) ; d \in\right.$ $(0, \infty)\}:=\Gamma_{k}$. (b) If $\left\{\left(\lambda_{n}, d_{n}\right)\right\}$ is a sequence in $\Gamma$ with $d_{n} \rightarrow \infty$, then $\left\{\lambda_{n}\right\}$ converges to $\mu_{k-1}$, where as if $\left\{d_{n}\right\}$ converges to zero, then $\left\{\lambda_{n}\right\}$ converges to $\mu_{k}$. (When $N=3$, then $\mu_{k-1}$ is to be replaced by $\left(k-\left(\frac{1}{2}\right)\right)^{2} \pi^{2}$.) (c) If $(\lambda, d) \in \Gamma_{k}$ then $\lambda<\mu_{k}$. (d) For each $k \geq 1$ there exists $q(k)$ such that if $(\lambda, d) \in \Gamma_{k}$, then $\lambda \geq q(k)$ and $q(k) \rightarrow \infty$ as $k \rightarrow \infty$. (e) $\{(\lambda, d) ; v(1, \lambda, d)=0, v(\cdot, \lambda, d)$ has exactly $k$ zeroes in $(0,1]\}$ is connected.

Proof. Part (a) follows directly from (5.6), Lemma 5.1, and the fact that the map $(\lambda, d) \rightarrow\left(\lambda, d \lambda^{1 / p}\right)$ defines a homeomorphism of $(0, \infty) \times(0, \infty)$ into itself.

Part (b) follows from part (a) and Lemma 5.1. 
In order to prove part (c) first we note that if $\Gamma$ is a connected component of $\{(\lambda, d) ; v(1, \lambda, d)=0\}$, then there exists a positive integer $k$ such that if $(\lambda, d) \in \Gamma$, then $v(\cdot, \lambda, d)$ has $k$ zeroes in $(0,1]$. By the Sturm comparison theorem we know that if $\lambda \geq \mu_{k}$, then $v(\cdot, \lambda, d)$ has at least $k$ zeroes in $(0,1)$. Hence, $\lambda \notin \Gamma$, and this proves part (c).

Since $y(r, \lambda, d)$ converges uniformly to zero on compact subsets of $(0, \infty)$, then for each $\lambda>0$ there exists $Q(\lambda)$ such that $v(r, \lambda, d)>Q(\lambda)$ for all $d \in\left(t_{1}, 1\right]$, where $t_{1}(d):=t_{1}$ is the first zero of $v(\cdot, \lambda, d)$. Because $E(\cdot, \lambda, d)$ is a decreasing function (see the definition of $E$ in (2.1)) we have $\lambda+|v(r, \lambda, d)|^{p}<Q(\lambda)$ for all $r \in\left[t_{1}, 1\right]$ and all $d>0$. Let $j$ be such that $\mu_{j}>Q(\lambda)$. By the Sturm comparison theorem it follows that $y(\cdot, \lambda, d)$ has at most $j+1$ zeroes in $(0,1]$. Hence $\beta_{k}(d)>\lambda$ for all $d>0$. Since $Q(\lambda) \rightarrow \infty$ as $\lambda \rightarrow \infty$, we see that $\min \beta_{k} \rightarrow \infty$ as $k \rightarrow \infty$, which proves part (d).

The proof of part (e) follows from the uniqueness of $\beta_{k}$, and that completes the proof of the lemma.

\section{Proof of Theorem 1.1 .}

(a) Let $\left\{d_{n}\right\}$ be such that $v\left(1, \lambda, d_{n}\right)=0$ for some $\lambda>0$. By part (d) of Lemma 5.2 there exists a positive integer $j$ such that $v\left(\cdot, \lambda, d_{n}\right)$ has at most $j$ zeroes in $(0,1]$. Thus, without loss of generality, we can assume that $v\left(\cdot, \lambda, d_{n}\right)$ has $k$ zeroes for each $n=1,2, \ldots$. By part b) of Lemma 5.2 we see that $\left\{d_{n}\right\}$ is a bounded sequence, unless $\lambda=\rho_{k}$ with $\rho_{k}=\mu_{k}(k=1,2, \ldots)$ if $N=4,5$ and $\rho_{k}=\left(k-\frac{1}{2}\right)^{2} \pi^{2}$ if $N=3$. This proves part (a) if $\lambda \neq \rho_{k}$.

Suppose $\lambda=\rho_{k}$. By Lemma 5.2 there exists $D>0$ such that if $v\left(1, \lambda_{0}, d\right)$ $=0, d>D$ and $\left|\lambda_{0}-\lambda\right|<\frac{1}{4}$, then $\left(\lambda_{0}, d\right) \in \Gamma_{k+1}$. From Lemma 4.2 without loss of generality, we can assume that $v_{d}\left(1, \lambda_{0}, d\right)>0$ and $v^{\prime}\left(1, \lambda_{0}, d\right)>0$. Hence, by the implicit function theorem there exists a differentiable function $s:(D, \infty) \rightarrow\left(\lambda-\frac{1}{4}, \lambda+\frac{1}{4}\right)$ such that $v\left(1, \lambda_{0}, d\right)=0$ iff $d=s\left(\lambda_{0}\right)$. Since $v_{\lambda}(1, s(d), d) \cdot s^{\prime}(d)+v_{d}(1, s(d), d)=0$ and $v_{d}(1, s(d), d) \neq 0$, we see that $s$ is strictly monotone. Thus, there exists at most one $n$ such $d_{n}>D$. Therefore, $\left\{d_{n}\right\}$ is bounded, which proves part (a).

(b) In order to prove part (b) it is sufficient to show that $s$ is a decreasing function. Let $\theta(r):=\theta(r, \lambda, d)$ denote the argument function such that

$$
v(r)=-\sqrt{v^{2}(r)+\left(v^{\prime}(r)\right)^{2}} \cos \theta(r), \quad v^{\prime}(r)=\sqrt{v^{2}(r)+\left(v^{\prime}(r)\right)^{2}} \sin \theta(r) .
$$

Differentiating with respect to $d$ and replacing $r$ by 1 we obtain

$$
v_{d}(1, \lambda, d)=v^{\prime}(1, \lambda, d) \cdot \theta_{d}(1, \lambda, d) .
$$

Hence by Lemma 4.2 we see that $\theta_{d}(1)>0$. Suppose that $s$ is not a decreasing function. Since $v(\cdot, s(d), d)$ has $k$ zeroes in $(0,1]$, we have $\theta(1, s(d), d)=$ $(k-1) \pi+\frac{\pi}{2}$. On the other hand, because $\theta_{d}(1, s(d), d)>0$ and $s$ is an increasing function we see that there exists $d^{\prime}>d$ such that $\theta\left(1, s(d), d^{\prime}\right)>$ $(k-1) \pi+\frac{\pi}{2}$. Since Pohozaev's identity (see Lemma 2.1) implies that $\theta(t, 0, d)$ $<\frac{\pi}{2}$ for all $t \in[0,1]$, by continuous dependence on parameters we see that for $\lambda$ small enough $\theta(1, \lambda, d)<\frac{\pi}{2}$. Therefore, by the intermediate value theorem, there exists $\lambda^{\prime}<s(d)$ such that $\theta\left(1, \lambda^{\prime}, d^{\prime}\right)=(k-1) \pi+\frac{\pi}{2}$. Thus, if $\left\{d_{n}\right\} \rightarrow \infty$ as $n \rightarrow \infty$, then there exists a sequence $\left\{\left(\lambda_{n}^{\prime}, d_{n}^{\prime}\right)\right\}$ such that 
$\theta\left(\lambda_{n}^{\prime}, d_{n}^{\prime}\right)=(k-1) \pi+\frac{\pi}{2}$. Hence, by Lemma 5.2 we see that $\left(\lambda_{n}^{\prime}, d_{n}^{\prime}\right)$ is in the connected component containing $(s(d), d)$. Thus, $\lambda_{n}=s\left(d_{n}\right)<s\left(d_{n}^{\prime}\right)=\lambda_{n}^{\prime}$, which contradicts that $\lambda_{n}^{\prime}<s\left(d_{n}^{\prime}\right)$. Therefore, $s$ is a strictly decreasing function.

(c) Let $\lambda_{0}>0$. Suppose that $v\left(1, \lambda_{0}, d\right)=0$ has infinitely many solutions $d_{1}, d_{2}, \ldots$. By part (a) we know that $\left\{d_{n}\right\}$ is bounded. Since $v\left(1, \lambda_{0}, d\right) \neq 0$ for $d$ large we see that $v\left(1, \lambda_{0}, \cdot\right)$ is not constant. Hence, because $v\left(1, \lambda_{0}, \cdot\right)$ is an analytic function (see Lemma 7.1) we have that $d_{n}$ converges to 0 . Thus $\lambda_{0}=\mu_{k}$ for some $k$ and $v\left(\cdot, \lambda_{0}, d_{n}\right)$ has $k$ zeroes in $[0,1]$. On the other hand, since $\lambda_{0}+|v|^{p}>\mu_{k}$ a.e., by the Sturm comparison theorem $v\left(\cdot, \lambda_{0}, d_{n}\right)$ has $k$ zeroes in $(0,1)$, hence $k+1$ zeroes in $(0,1]$, which is a contradiction. Hence, $v\left(1, \lambda_{0}, \cdot\right)$ has finitely many zeroes. Thus, the theorem is proven.

\section{APPENDIX}

Our next lemma, to be used for the case $N=3,4$, can be viewed as a version of the classical Cauchy-Kowalewskaya theorem (see [11]) for singular equations. For the sake of completeness we include a sketch of the proof.

Lemma 7.1. For $N=3,4$, the function $v(t, \lambda, \cdot)$ is real analytic on $(-\infty, 0)$ $\cup(0, \infty)$.

Proof. Since $v(t, \lambda, \cdot)$ is an odd function it is sufficient to show that for each $\hat{\delta} \in(0, \infty)$ there exists $\eta>0$ and a sequence $\left\{v_{j}(t, \lambda)\right\}$ such that if $|d-\hat{\delta}|<\eta$ then

$$
v(t, \lambda, d)=\sum_{j=0}^{\infty} v_{j}(t, \lambda)(d-\hat{\delta})^{j} / j !
$$

Let $v_{0}(t, \lambda)=v(t, \lambda, \hat{\delta})$. Since $\frac{d E}{d t} \leq 0$, we have that $\left|v_{0}(t, \lambda)\right| \leq \hat{\delta}$. We define $v_{1}(t):=v_{1}(t, \lambda)$ as the solution to

$$
\begin{gathered}
v_{1}^{\prime \prime}(r)+\frac{N-1}{r} v_{1}^{\prime}(r)+\lambda v_{1}(r)+(p+1)\left|v_{0}(r)\right|^{p} v_{1}(r)=0, \\
v_{1}(0)=1, \quad v_{1}^{\prime}(0)=0
\end{gathered}
$$

(if $N=3,4$ then $p=4,2$ respectively). Inductively for $k \geq 1$ we define $v_{k+1}(t)$ as the solution to

$$
\begin{aligned}
& v_{k+1}^{\prime \prime}(r)+\frac{N-1}{r} v_{k+1}^{\prime}(r) \\
& +\lambda v_{k+1}(r)+(p+1)\left|v_{0}(r)\right|^{p} v_{k+1}(r)+\omega_{k+1}(r)=0, \\
& v_{k+1}(0)=0, \quad v_{k+1}^{\prime}(0)=0,
\end{aligned}
$$

where

$$
\omega_{k+1}=\sum_{\substack{i_{0}+\cdots+i_{p}=k+1 \\
k \geq i_{0}, \ldots, i_{p} \geq 0}}\left(\begin{array}{c}
k+1 \\
i_{0}
\end{array}\right)\left(\begin{array}{c}
k+1-i_{0} \\
i_{1}
\end{array}\right) \cdots\left(\begin{array}{c}
k+1-i_{0}-\cdots-i_{p-2} \\
i_{p-1}
\end{array}\right) v_{i_{0}} \cdots v_{i_{p}} .
$$


Since $v_{k+1}$ is given by

$$
v_{k+1}(t)=-\int_{0}^{t} r^{1-N} \int_{0}^{r} s^{N-1}\left\{\left[\lambda+(p+1)\left|v_{0}(s)\right|^{p}\right] v_{k+1}(s)+\omega_{k+1}(s)\right\} d s d r,
$$

we see that if $t \in\left[0, a:=\left(N /\left(\lambda+(p+1) \hat{\delta}^{p}\right)\right)^{1 / 2}\right]$, then

$$
\max _{0 \leq t \leq a}\left|v_{k+1}(t, \lambda)\right|=\left|v_{k+1}\left(t^{0}\right)\right| \leq 2 \int_{0}^{t} r^{1-N} \int_{0}^{r} s^{N-1}\left|\omega_{k+1}(s)\right| d s d r,
$$

where, of course $t^{0} \in[0, a]$ depending on $k$. Now, imitating the arguments of [11, Lemmas 2.2, 2.3, and 2.4] it can be shown that

$$
\left|v_{k+1}(t, \lambda)\right|+\left|\omega_{k+1}(t, \lambda)\right| \leq \frac{C^{k+1}(k+1) !}{(k+1)^{2}},
$$

where $C$ is a constant independent of $k$. Hence, the series in (7.1) defines an analytic function in $d$. In order to prove the validity of (7.1) we show that the power series in (7.1) is twice differentiable with respect to $t$, and satisfies (1.2). Indeed, from (7.3) we have

$$
\begin{aligned}
\left|v_{k+1}^{\prime}(t)\right| & =\left|-t^{1-N} \int_{0}^{t} s^{N-1}\left[\lambda+(p+1)\left|v_{0}(s)\right|^{p}\right] v_{k+1}(s)+\omega_{k+1}(s) d s\right| \\
& \leq t C^{k+1} \frac{(k+1) !}{(k+1)^{2}}
\end{aligned}
$$

From (7.2), (7.4), and (7.5) we obtain

$$
\left|v_{k+1}^{\prime \prime}(t)\right| \leq\left(N+\lambda+(p+1) \hat{\delta}^{p}\right) C^{k+1} \frac{(k+1) !}{(k+1)^{2}} .
$$

Thus

$$
\sum_{j=0}^{\infty} v_{j}^{\prime}(t, \lambda)(d-\hat{\delta})^{j} / j !, \quad \sum_{j=0}^{\infty} v_{j}^{\prime \prime}(t, \lambda)(d-\hat{\delta})^{j} / j !, \quad \sum_{j=0}^{\infty} v_{j}^{\prime}(t, \lambda)(d-\hat{\delta})^{j} / t j !,
$$

converge. Since

$$
\left(\sum_{j=0}^{\infty} v_{j}(t, \lambda)(d-\hat{\delta})^{j} / j !\right)^{\prime}=\sum_{j=0}^{\infty} v_{j}^{\prime}(t, \lambda)(d-\hat{\delta})^{j} / j !,
$$

and similarly for the second derivative with respect to $t$, from (7.2) we obtain

$$
\begin{aligned}
\sum_{j=0}^{\infty} v_{j}^{\prime \prime}(t)(d-\hat{\delta})^{j} / j !+ & \sum_{j=0}^{\infty} \frac{N-1}{t} v_{j}^{\prime}(t)(d-\hat{\delta})^{j} / j !+\sum_{j=0}^{\infty} \lambda v_{j}(t)(d-\hat{\delta})^{j} / j ! \\
& +\left(\sum_{j=0}^{\infty} v_{j}(t)(d-\hat{\delta})^{j} / j !\right)^{p+1}
\end{aligned}
$$




$$
\begin{aligned}
= & \sum_{j=0}^{\infty} v_{j}^{\prime \prime}(t)(d-\hat{\delta})^{j} / j !+\sum_{j=0}^{\infty} \frac{N-1}{t} v_{j}^{\prime}(t)(d-\hat{\delta})^{j} / j !+\sum_{j=0}^{\infty} \lambda v_{j}(t)(d-\hat{\delta})^{j} / j ! \\
& +\sum_{j=0}^{\infty}\left[(p+1)\left|v_{0}(t)\right|^{p} v_{j}(t)+\omega_{j}(t)\right](d-\hat{\delta})^{j} / j ! \\
= & \sum_{j=0}^{\infty}\left[v_{j}^{\prime \prime}(t)+\frac{N-1}{t} v_{j}^{\prime}(t)+\lambda v_{j}(t)+(p+1)\left|v_{0}(t)\right| v_{j}(t)+\omega_{j}(t)\right](d-\hat{\delta})^{j} / j ! \\
= & 0 .
\end{aligned}
$$

Also, for all $d \in(\hat{\delta}-\eta, \hat{\delta}+\eta)$ we have

$$
\sum_{j=0}^{\infty} v_{j}(0)(d-\hat{\delta})^{j} / j !=\hat{\delta}+d-\hat{\delta}=d
$$

and

$$
\sum_{j=0}^{\infty} v_{j}^{\prime}(0)(d-\hat{\delta})^{\gamma} / j !=0
$$

Hence (7.1) holds. Since the differential equation in (1.2) is regular and analytic on $[a, 1]$ we see that the transformation $\left(v(a, \lambda, d), v^{\prime}(a, \lambda, d)\right) \rightarrow$ $\left(v(1, \lambda, d), v^{\prime}(1, \lambda, d)\right)$ is an analytic function of $d$. Thus, the map $d \rightarrow$ $\left(v(1, \lambda, d), v^{\prime}(1, \lambda, d)\right)$ is analytic, which proves the lemma.

\section{REFERENCES}

1. F. Atkinson, H. Brezis, and L. Peletier, Solutions d'equations elliptiques avec exposant de Sobolev critique qui changent de signe, C.R. Acad. Sci. Paris Ser. I Math. 306 (1988), 711714.

2. H. Brezis and L. Nirenberg, Positive solutions of nonlinear elliptic equations involving critical Sobolev exponents, Comm. Pure Appl. Math. 36 (1983), 437-477.

3. A. Castro and A. Kurepa, Infinitely many radially symmetric solutions to a superlinear Dirichlet problem in a ball, Proc. Amer. Math. Soc. 101 (1987), 56-64.

4. A. Castro and A. Kurepa, Radially symmetric solutions to a superlinear Dirichlet problem in a ball with jumping nonlinearities, Trans. Amer. Math. Soc. 315 (1989), 353-372.

5. G. Cerami, Elliptic equations with critical growth, College on Variational Problems in Analysis, Lecture Notes SMR 281/24, Internat. Centre for Theoretical Physics, Trieste, Italy, 1988.

6. G. Cerami, S. Solimini, and M. Struwe, Some existence results for superlinear elliptic boundary value problems involving critical exponents, J. Funct. Anal. 69 (1986), 289-306.

7. M. G. Crandall, and P. M. Rabinowitz, Bifurcation from simple eigenvalues, J. Funct. Anal. 8 (1971), 321-340.

8. M. K. Kwong, Uniqueness of positive solutions for $\Delta u-u+u^{p}=0$ in $R^{n}$, Arch. Rational Mech. Anal. 105 (1989), 243-266.

9. S. I. Pohozaev, Eigenfunctions of the equation $\Delta u+\lambda f(u)=0$, Soviet. Math. Dokl. 6 (1965), 1408-1411.

10. P. Pucci and J. Serrin, A general variational identity, Indiana Univ. Math. J. 35 (1986), 681-703.

11. M. Shinbrot and R. Welland, The Cauchy-Kowalewskaya theorem, J. Math. Anal. Appl. 55 (1976), 757-772. 
12. S. Solimini, On the existence of infinitely many radial solutions for some elliptic problems, Rev. Mat. Apl. 9 (1987), 75-86.

13. N. Trudinger, Remarks concerning the conformal deformation of Riemannian structures on compact manifolds, Ann. Sci. Norm. Sup. Pisa 22 (1968), 265-274.

Department of Mathematics, University of North Texas, Denton, Texas 76203-5116

Department of Mathematics, North Carolina A \& T University, Greensboro, North CAROLINA 27411

E-mail address: kurepancat.edu 